\title{
O PROGRAMA DE APRENDIZAGEM SOCIOEMOCIONAL - ATITUDE POSITIVA 3 CICLO NAS ACADEMIAS GULBENKIAN DO CONHECIMENTO
}

\author{
Marta Marchante \\ Académico de Torres Vedras \\ marta.isabelmarchante@gmail.com \\ Vítor Alexandre Coelho \\ Académico de Torres Vedras \\ Vitorpcoelho@gmail.com
}

Recepción Artículo: 10 mayo 2021

Admisión Evaluación: 10 mayo 2021

Informe Evaluador 1: 18 mayo 2021

Informe Evaluador 2: 26 mayo 2021

Aprobación Publicación: 01 junio 2021

\begin{abstract}
RESUMO
Durante os últimos 20 anos têm crescido exponencialmente as iniciativas que promovem 0 desenvolvimento de competências socioemocionais sob a égide da moldura teórica do Collaborative for Academic, Social and Emotional Learning. No entanto, o desenvolvimento de programas de aprendizagem socioemocional em Portugal tem sido consistente (Cristovão et al., 2017), mas lento. Uma análise independente sobre o desenvolvimento de programas de aprendizagem socioemocional em Portugal identificou os programas Atitude Positiva como um dos programas com maior validação científica em Portugal (Cristovão et al., 2017). Em 2019, a Fundação Calouste Gulbenkian apresentou uma iniciativa, as Academias Gulbenkian do Conhecimento (AGC) para promover 0 desenvolvimento de iniciativas que promoviam o desenvolvimento de competências socioemocionais em Portugal. Como parte desta iniciativa, as AGC financiavam replicações de alguns programas descritos como de referência, um dos quais o programa de aprendizagem socioemocional - Atitude Positiva $3^{0}$ ciclo. Consequentemente, o programa de aprendizagem socioemocional - Atitude Positiva $3^{\circ}$ Ciclo foi selecionado para implementação em 6 Academias Gulbenkian do Conhecimento distribuídas por todo o território de Portugal. Assim, o presente manuscrito descreve as sucessivas fases de seleção de entidades que procuravam replicar o programa, descreve o processo de formação, a metodologia de avaliação e a forma de controlar a fidelidade de implementação e outros instrumentos que foram criados para apoiar a replicação do programa de aprendizagem socioemocional - Atitude Positiva 3ํㅡㄴ Ciclo. 0 manuscrito identifica ainda as conclusões que são passíveis de retirar deste processo.
\end{abstract}




\section{O PROGRAMA DE APRENDIZAGEM SOCIOEMOCIONAL - ATITUDE POSITIVA $3^{\circ}$ CICLO NAS ACADEMIAS GULBENKIAN DO CONHECIMENTO}

Palavras-chave: competências socioemocionais; disseminação de programas; fidelidade de implementação

\section{ABSTRACT}

The atitude positive $-3^{\circ}$ ciclo social and emotional learning program a part of the gulbenkian academies of knowledge. [During the last 20 years there has been an exponential growth in initiatives that promote the development of social and emotional competencies under the guidance of the Collaborative for Academic, Social and Emotional Learning. However, the development of social and emotional learning programs in Portugal has been consistent (Cristovão et al., 2017), but slow. An independent analysis regarding the development of social and emotional learning programs in Portugal has concluded the the Atitude Positiva programs can be considered as among the programs with more scientific validation in Portugal (Cristovão et al., 2017). In 2019, the Calouste Gulbenkian Fundation created an iniciative, the Gulbenkian Academies of Knowledge to promote the creation and/or dissemination of initiatives for the development of social and emotional competencies in Portugal. As part of this iniciative, the Gulbenkian Foundation financed the replication of some blueprint programs, one of which was the Atitude Positiva - $3^{\circ}$ ciclo SEL program. Therefore, this program was selected for implementation in six Gulbenkian Academies of Knowledge distributed all throughout Portugal. This, the present manuscript describes the phases of selection of the entities which sought to replicate the program, it also details the formation process, the assessment methodology, and how the implementation fidelity was controlled during the replication of the Atitude Positiva $3^{\circ}$ Ciclo SEL program. The manuscript also identifies the conclusion that are possible to draw from this process.]

Keywords: social and emotional learning; replication program; fidelity and monitorization

\section{INTRODUÇÃO}

Durante os últimos 15 anos tem aumentado as evidências de que o desenvolvimento de competências socioemocionais é crucial para a adaptação das crianças às exigências da sociedade, de forma a adaptarem-se às necessidades complexas do crescimento e desenvolvimento (Greenberg el al., 2017; Weissberg et al., 2015). Aliás, dentro da área da promoção da saúde mental, Knapp et al. (2011) num estudo da London Business School of Economics apresentavam mesmo o desenvolvimento de competências socioemocionais como uma das intervenções que apresenta um melhor rácio custo/benefício. Nesta área destaca-se a Aprendizagem SocioEmocional (Weissberg et al., 2015), abordagem que conta com mais de 500 avaliações de programas neste domínio. Adicionalmente, várias meta-análises têm identificado que os programas Social and Emotional Learning (SEL) de carácter universal, contribuem para melhorias no ambiente escolar, rendimento académico, comportamento pró-social, atitudes face ao próprio e à escola (Durlak et al. 2011; Sklad et al., 2012; Taylor et al., 2017). Por exemplo, atualmente nos EUA milhares de escolas implementam programas SEL, desde a Educação Pré-Escolar até ao Ensino Superior, sendo obrigatórios em alguns estados americanos (Weissberg et al., 2015).

0 Projeto Atitude Positiva é desenvolvido pelo Académico de Torres Vedras no concelho de Torres Vedras há 17 anos e há três anos no concelho do Cadaval. Trata-se de um projeto de prevenção com enfoque na promoção de competências socioemocionais cujos principais objetivos são a promoção de estilos de vida saudáveis, a prevenção de comportamentos de risco e promover integração escolar. Todos estes objetivos são promovidos através do desenvolvimento das competências socioemocionais dos alunos.

As atividades principais do Projeto Atitude Positiva incluem Programas de Aprendizagem SocioEmocional para alunos do $1^{\circ}, 2^{\circ}$ e $3^{\circ}$ ciclo; Programas de Ajustamento Escolar de $1^{\underline{0}}$ para $02^{\circ}$ 
ciclo e de $2^{0}$ para $03^{0}$ ciclo (Transição Positiva); Ações de Informação e Sensibilização para alunos; um Programa de igualdade de género para alunos de $2^{0}$ e 3 ciclos. Ainda com os alunos realizamse Acompanhamentos Psicológicos Individuais e Tutorias Psicopedagógicas. Adicionalmente, são realizadas Oficinas de Formação Assistentes Operacionais e Encarregados de Educação.

Programa de Aprendizagem Socioemocional - 3ํㅗㄷㅇ

Este programa tem como principais objetivos o desenvolvimento de competências socioemocionais que estão identificadas como protetoras de estilos de vida saudáveis e como fatores influentes numa integração mais efetiva na vida escolar. Neste sentido, promove-se o desenvolvimento saudável e pleno das crianças e jovens, sendo esperado que se tornem mais confiantes e competentes para lidarem com os desafios do quotidiano, promovendo-se a integração e, consequentemente, o sucesso escolar. 0 programa é desenvolvido pelo psicólogo que orienta o projeto em cada agrupamento e é constituído por atividades que implicam o relacionamento interpessoal, através de jogos e outras dinâmicas. Os principais temas abordados são a comunicação, o trabalho em equipa, a gestão de conflitos, autoestima, entre outros. A estrutura geral do programa assenta em 13 sessões (45 a 50 minutos), que inclui dois módulos, avaliação inicial e final. Para se proceder à avaliação do programa, é necessária a colaboração do docente através de uma reunião e do preenchimento de questionários relativos aos alunos, no início e no final do programa.

0 programa de Aprendizagem SocioEmocional - Atitude Positiva ( $3^{\circ}$ ciclo) tem sido validado através de diversos estudos de eficácia e efetividade do programa. Um primeiro estudo, de Coelho et al. (2017), com 628 participantes, incidiu sobre o impacto do programa nas competências socioemocionais durante os dois primeiros anos de implementação do programa. Observou-se um aumento significativo na Consciência Social e no Autocontrolo, bem como uma diminuição significativa na Ansiedade Social relatada pelos alunos que participaram no programa, relativamente aos que não participaram. Foram também encontrados resultados diferenciais por género, com as raparigas a revelarem maiores ganhos na Consciência Social, Isolamento Social e Ansiedade Social face aos rapazes. Os alunos que apresentavam níveis iniciais mais baixos de Consciência Social beneficiaram mais da participação no programa. Num segundo estudo (Coelho, Marchante \& Sousa, 2015), com 1091 participantes, foram analisados os três anos seguintes de implementação do programa, estudando se o seu impacto, sobre as competências socioemocionais, relatadas pelos alunos e pelos seus respetivos professores. Neste estudo observaram se resultados positivos nos relatos dos alunos em cinco das seis variáveis analisadas: na Consciência Social, no Autocontrolo, no Isolamento Social, na Ansiedade Social e na Autoestima. Os dados fornecidos pelos professores demonstraram resultados positivos em todas as cinco dimensões analisadas.

Adicionalmente, Coelho e Sousa em 2017 analisaram o impacto de variáveis de nível classe na efetividade do programa de aprendizagem socioemocional de $3^{\circ}$ ciclo ( $7^{\circ}$ ao $9^{\circ}$ ano) reportados pelos alunos e sus professores. 0 estudo consistia em 746 alunos (576 no grupo de intervenção e 170 no grupo de controlo). Os resultados, de auto e hetero-relato, identificam ganhos positivos na consciência social e autocontrolo. Os alunos de escolas rurais parecem ter ganhos superiores na consciência social.

Ainda em 2018, Coelho e Sousa analisaram o impacto das diferenças no setting de implementação nos resultados obtidos no programa de aprendizagem socioemocional. 0 estudo comparava a efetividade do programa aplicado em horário escolar e pós letivo. Participaram 837 alunos e os resultados de análises multinível identificaram impactos mais positivos na autoestima, autocontrolo e consciência social nos grupos que funcionavam em horário escolar.

Outros estudos analisaram o impacto do programa nas várias dimensões do Autoconceito dos alunos. Um estudo transversal (Coelho, Sousa, \& Figueira, 2014), envolvendo 2 anos escolares, 
com 630 participantes, permitiu observar um aumento significativo no Autoconceito Social, Emocional e Autoconceito total, dos participantes no programa relativamente ao grupo de controlo. Indicou também que alunos com baixos níveis de competências iniciais (Autoconceito Académico e Social), beneficiaram mais da sua participação no programa. Foram ainda observados efeitos de género na dimensão do Autoconceito Emocional, na qual apenas os rapazes apresentaram ganhos. Também em 2016, um estudo com 2682 alunos analisou o impacto diferencial das intervenções no autoconceito entre alunos de primeiro e terceiro ciclo. Os resultados reforçam o impacto do programa no Autoconceito Social e Emocional (Coelho, Marchante e Sousa, 2016).

Avaliação do Programa SocioEmocional - Atitude Positiva (3ํㅜㄷㄴ)

0 Projeto Atitude Positiva desenvolveu um conjunto de instrumentos de avaliação das competências socioemocionais pois a avaliação adotada entre 2005 e 2009 apresentava um conjunto de fragilidades:

1. Discrepância de dimensões entre 0 instrumento de autorrelato e o de hétero relato;

2. Desfasamento entre o instrumento de autorrelato e a moldura teórica adotada, nomeadamente pela ausência de escalas que avaliassem competências relacionais e tomada de decisão responsável;

3. Críticas espontâneas dos estudantes de $3^{0}$ ciclo (7ํa aํano) face à extensão do instrumento de autorrelato e, particularmente, relativamente ao seu formato de resposta (dicotómica: Sim ou Não) que classificavam como extremamente limitativa e que consideravam não Ihes permitir expressar a sua realidade.

Desta forma, surgiu a necessidade de elaborar um sistema que apresentasse uma maior correspondência com a base teórica proposta pela CASEL (2005), que incluísse formas coordenadas para diferentes informantes (autorrelato por alunos e hétero relato por professores) e que permitisse a avaliação de um conjunto de programas de aprendizagem socioemocional portugueses. Assim, foram desenvolvidos e validados dois instrumentos que permitem a avaliação das competências socioemocionais e dos programas de Aprendizagem SocioEmocional, de acordo com o modelo proposto pela CASEL (2005), através de dois informantes, os alunos e os seus professores.

Estes instrumentos foram criados para sejam usados na avaliação da eficácia e efetividade de programas de Aprendizagem SocioEmocional, tendo sido baseados em três instrumentos: Bateria de Socialización 1 (Silva \& Martorell, 2001), Social Skills Rating Scale (Gresham \& Elliott, 1990) e o School Social Behavior Rating Scale (Merrell, 2002), amplamente utilizados em contexto ibérico e maioritariamente adaptados para a população portuguesa, mas que não correspondiam às exigências do projeto, nomeadamente, devido à sua baixa correspondência ao modelo teórico adotado.

Academias Gulbenkian do Conhecimento

0 PASE $3^{\circ}$ Ciclo foi selecionado como metodologia de referência para as Academias Gulbenkian do Conhecimento. Desta forma, passou a ser elegível para que várias entidades se pudessem candidatar à sua replicação. Durante a primeira fase de seleção candidataram-se 24 entidades. A Fundação Calouste Gulbenkian realizou uma avaliação e passaram à segunda fase 9 entidades. Após uma nova fase de avaliação, foram selecionadas 5 Academias para replicarem 0 Programa de Aprendizagem SocioEmocional - Atitude Positiva (3ํㅡㄴ Ciclo): Vizela ImaginAtiva (Vizela) Associação de Pais do Pombal (Pombal), ANADIC (Lisboa e Caniçal - Madeira), Associação Cristã da Mocidade de Setúbal - ACM (Setúbal) e Potencialmente Algarve (Algarve).

\section{OBJETIVOS DA INVESTIGAÇÃO}

0 presente manuscrito pretende analisar o processo de formação das academias e o modelo de monitorização desenvolvido no âmbito da replicação do programa de Aprendizagem SocioEmocional para $03^{\circ}$ ciclo - Atitude Positiva. 


\section{PARTICIPANTES}

Neste estudo participaram 6 Academias (já que na prática as Academias da Madeira e de Lisboa da ANADIC funcionavam como independentes). Cada Academia identificou os técnicos (que tinham obrigatoriamente de deterem formação superior em Psicologia) que iriam os implementadores do programa. Estes técnicos realizaram a formação inicial, num total de 16 técnicos.

Adicionalmente, as academias identificaram um responsável/coordenador, que participou na formação onsite sobre a plataforma e acompanhou o processo de monitorização (introdução de dados na plataforma).

No segundo ano de replicação do programa (que não é objeto de análise neste manuscrito), devido à situação pandémica mundial, a formação inicial dos 2 novos técnicos decorreu totalmente em formato online.

\section{METODOLOGIA \\ Procedimento}

A formação dos técnicos e responsáveis das Academias Gulbenkian do Conhecimento foi programada de forma a englobar uma fase inicial de formação em pequeno grupo e uma segunda fase formação onsite na Academia e apenas com os técnicos de cada Academa.

A formação inicial, foi ministrada em dois grupos, tendo ambos decorrido em Setembro de 2019. Cada formação consistiu em 4 dias com $7 \mathrm{~h}$ de formação presencial perfazendo um total de 28h. A formação seguiu um modelo teórico-prático, fornecendo as bases teóricas para uma melhor compreensão do modelo de aprendizagem socioemocional, assim como formação sobre a avaliação do programa. Por outro lado, pretendia também dotar os formandos das ferramentas necessárias para aplicar as várias atividades do programa, e como tal, os técnicos foram convidados a experimentar as várias atividades enquanto participantes, mas também enquanto aplicadores de algumas atividades. Os objetivos desta formação foram organizados da seguinte forma: no primeiro dia pretendia-se que os formandos compreendessem os conceitos de Aprendizagem SocioEmocional, a perpectiva de evolução desta abordagem nos últimos 20 anos, SEL em Portugal e no Mundo. No segundo dia de formação os formandos ficaram a conhecer o projeto Atitude Positiva e 0 enquadramento do programa de Aprendizagem SocioEmocional de $3^{\circ}$ ciclo. No terceiro dia o foco esteve em conhecer o Programa de Aprendizagem SocioEmocional Atitude Positiva - $3^{0}$ ciclo, os seus módulos, objetivos e atividades, contando com uma abordagem de experimentação de algumas atividades. Por fim, no quarto dia de formação, os formandos puderam identificar os principais princípios da Avaliação de Programas de Aprendizagem SocioEmocional, os instrumentos utilizados no programa e a sua aplicação.

Para a avaliação da eficácia do programa, são aplicados os questionários Questionário de Avaliação de Competências SocioEmocionais e Self-Description Questionnaire-II aos alunos após a sessão de apresentação (avaliação inicial) e após o término das atividades dos 2 módulos (avaliação final). Simultaneamente, no mesmo período de forma a avaliar os mesmos momentos, são aplicados os questionários Questionário de Avaliação de Competências SocioEmocionais-Professores (versão reduzida) aos professores (avaliação inicial e final).

De forma a garantir que os resultados observados nos grupos de intervenção fossem atribuídos à intervenção e não a outros fatores, foi recomendado que existissem grupos de controlo, com características semelhantes às turmas de intervenção.

Para efeito da avaliação da efetividade da aplicação do programa de Aprendizagem SocioEmocional - Atitude Positiva ( $3^{\circ}$ Ciclo) apenas foram incluídos os alunos que apresentavam, concomitantemente avaliação inicial e avaliação final. 0 mesmo critério foi utilizado relativamente à avaliação pelos professores. 


\section{O PROGRAMA DE APRENDIZAGEM SOCIOEMOCIONAL - ATITUDE POSITIVA $3^{\circ}$ CICLO NAS ACADEMIAS GULBENKIAN DO CONHECIMENTO}

\section{Instrumentos}

Questionário de Avaliação de Competências Socioemocionais (QACSE; Coelho, Sousa e Marchante, 2015; Coelho e Sousa, 2016). 0 QACSE, é um instrumento de autorrelato desenvolvido para avaliar as competências socioemocionais de adolescentes dos 12 aos 16 anos, presentemente composto por 39 itens, a responder numa escala de resposta de quatro pontos ( $A=N$ Nunca; $B=$ Algumas vezes; $C=$ Frequentemente; $D=S e m p r e)$, organizados em seis dimensões, cada uma das quais com 7 itens, com a exceção da escala de Tomada de Decisão Responsável que é composta por 4 itens:

1. Autocontrolo (ex.: "Quando quero falar, peço a palavra e espero pela minha vez."); ( =.73);

2. Consciência Social (ex.: "Fico preocupado/a quando alguém tem problemas."); (=.87)

3. Competências Relacionais (ex.: "Sou popular entre o/as outros/as."); ( =.71)

4. Tomada de Decisão Responsável (ex.: "Quando preciso de tomar uma decisão pondero as consequências."); ( =.76)

5. Isolamento Social (ex.: "Isolo me e não falo com ninguém."); ( =.74)

6. Ansiedade Social (ex.: "Sou envergonhado."), ( =.78)

0 instrumento já foi validado em dois estudos. Num primeiro estudo realizado por Coelho, Sousa e Marchante (2015), com 683 participantes, foi realizada uma Análise Fatorial Exploratória que isolou cinco fatores que explicavam $42.2 \%$ da variância. As subescalas, de acordo com os critérios estabelecidos por Cicchetti (1994), apresentavam valores de consistência interna adequados e de estabilidade temporal. Posteriormente, num segundo estudo com 655 alunos de $3^{\circ}$ ciclo foi incluído no instrumento uma escala de Tomada de Decisão Responsável, e foi realizada uma Análise Fatorial Confirmatória, que confirmou a estrutura fatorial composta por 6 dimensões. Desta forma, o questionário, na sua presente forma, cumpre a função de ser um instrumento curto, eficiente, com um formato de respostas mais discriminativo e com maior correspondência às outras medidas desenvolvidas pelo projeto e ao referencial da CASEL (2005).

No âmbito das Academias do Conhecimento Gulbenkian, para avaliar a efetividade da implementação do Programa de Desenvolvimento SocioEmocional - Atitude Positiva (3ํㅡㄷㄷㅇ)

Questionário de Avaliação de Competências Socioemocionais - versão professores/forma reduzida (QACSE P-SF; Coelho, Sousa e Marchante, 2014, 2016). 0 QACSE P-SF é presentemente composto por 30 itens, com 5 itens por escala, e é um instrumento de hetero-relato que avalia a percepção dos professores relativamente às competências socioemocionais dos seus alunos. Utiliza a mesma escala de resposta do instrumento de autorrelato e requer que o professor despenda 5 minutos por aluno no seu preenchimento.

1. Autocontrolo (ex:: "Quando quer falar, espera pela sua vez."); ( = .84),

2. Consciência Social (ex.: "Fica preocupado/a quando alguém tem problemas."); ( =.81)

3. Competências relacionais (ex.: "É capaz de discutir regras que lhe pareçam injustas."); (=.92

4. Isolamento Social (ex.: "Isola se e não fala com ninguém."); ( =.91)

5. Ansiedade Social (ex.: "Tem medo perante situações ou atividades novas."); ( =.84)

6. Tomada de decisão responsável (ex.: "Considera várias alternativas antes de tomar uma decisão"); ( =.85)

As subescalas, de acordo com os critérios estabelecidos por Cicchetti (1994), apresentavam bons valores de consistência internas; as análises realizadas apoiaram também a validade concorrente e convergente do instrumento (Coelho et al., 2014, 2015).

Num primeiro estudo de validação do questionário (Coelho, Sousa e Marchante, 2014), 0 QACSE-P, foi validado junto de 79 professores do distrito de Lisboa (sobre 1214 alunos do $4^{\circ}$ ao 9o 
ano de escolaridade de escolas rurais e urbanas). Esta versão do QACSE-P era composta por 56 itens, organizados em 5 escalas, que no geral reproduzia competências socioemocionais identificadas no modelo de aprendizagem socioemocional preconizado pelo CASEL Neste estudo foi realizada uma análise fatorial exploratória, que identificou cinco fatores que explicavam $57.9 \%$ da variância. No geral, os resultados deste estudo sugeriam a existência de boas caraterísticas psicométricas do QACSE-P, apresentando uma estrutura fatorial adequada, bons índices de consistência interna, estabilidade teste-reteste e bons indicadores de validade convergente e divergente (Coelho, Sousa e Marchante, 2014). No entanto, a pedido dos professores que consideravam esta versão muito longa, foi realizado um segundo estudo de validação (Coelho, Sousa e Marchante, 2016), desta vez para uma versão reduzida do questionário. Neste estudo, 39 professores completaram o questionário sobre 657 alunos ( $4^{\circ}$ ao $9^{\circ}$ ano). Uma análise fatorial suportou a estrutura de 6 fatores que explicavam $67.6 \%$ da variância. Para além da redução do número de itens do instrumento, esta versão incluiu também uma escala de Tomada de Decisão Responsável, o que permitiu um maior alinhamento com a moldura teórica da CASEL. Finalmente, em 2016 foi realizado um terceiro estudo, em que 75 professores avaliaram 1258, onde foi realizada uma Análise Fatorial Confirmatória que permitiu confirmar a estrutura fatorial do instrumento.

Autoestima. A autoestima foi avaliada através da versão portuguesa (Fontaine, 1991) da escala global de Autoestima do Self -Description Questionnaire II (Marsh et al., 1983). A escala permite a avaliação da autoestima de uma forma global, sendo composta por 10 itens (ex.: "Em geral, tenho muito do que me orgulhar") avaliados numa escala de cinco pontos (1=Falso; 2=Frequentemente falso; $3=$ Nem verdadeiro nem falso; 4=Frequentemente verdadeiro e 5=Verdadeiro); cinco dos quais são apresentados de forma negativa. A consistência interna da escala foi adequada para ambas as versões (original e adaptação Portuguesa; $=.88$ e .82, respetivamente).

Satisfação. A satisfação com o programa foi avaliada através de um questionário de nove itens (Coelho, Freitas \& Sousa, 2006), cinco deles avaliados numa escala de cinco pontos (ex.: "Achas que as sessões foram" de 1=muito más a $5=$ =muito boas) e outros quatro itens qualitativos (ex.: "Gostarias de participar outra vez? Porquê?").

\section{RESULTADOS}

Após a formação foi realizado um questionário anónimo de avaliação da mesma, no sentido de perceber quais as áreas/temas de maior/menor satisfação por parte dos formandos. As questões colocadas apresentavam uma escala de resposta de 1 a 5 (1 - Discordo totalmente, 2 - Discordo, 3 -Nem concordo nem discordo, 4 - Concordo, 5 - Concordo totalmente). Na tabela 1 podemos confirmar as médias das respostas dos formandos às questões colocadas, verificando-se uma elevada satisfação com a participação na formação. Dentro dos pontos fortes da formação, foi salientado "conhecer mais acerca de ASE e ver um programa integrado, com validade científica comprovada" e maioritariamente 0 caráter prático da formação e a possibilidade de realizar as dinâmicas de grupo.

Os aspetos da formação com os quais os formandos demonstram menor apreciação foram 0 tema da estatística, a falta de interação entre grupos de formação e a distância percorrida para participar da formação.

Ainda no questionário de avaliação da formação, os técnicos foram questionados sobre os temas que gostariam de ver abordados na próxima formação. Diversas sugestões sobre a avaliação (aplicação e tratamento dos dados, técnicas de análise de dados, estabelecimento do perfil das turmas), formação sobre a plataforma, assim como pedido de formação nos outros programas do projeto. Adicionalmente foi identificada a necessidade de supervisão, incluindo exercícios práticos como role-plays e autoescopias. 


\section{O PROGRAMA DE APRENDIZAGEM SOCIOEMOCIONAL - ATITUDE POSITIVA $3^{\circ}$ CICLO NAS ACADEMIAS GULBENKIAN DO CONHECIMENTO}

Tabela 1 - Resultados da avaliação de satisfação com a formação pelos técnicos das Academias

\begin{tabular}{llll}
\hline & Média & Mínimo & \multicolumn{2}{c}{ Máximo } \\
\hline Aumento de conhecimentos & 4.8 & 4 & 5 \\
Tempo disponível & 4.47 & 4 & 5 \\
Dificuldade das atividades & 4.73 & 4 & 5 \\
Material disponibilizado & 4.87 & 4 & 5 \\
Técnicas utilizadas & 4.6 & 4 & 5 \\
Clareza dos formadores & 4.73 & 4 & 5 \\
Estabelecimento de dinâmicas positivas & 4.67 & 4 & 5 \\
Boa relação com os formadores & 4.87 & 4 & 5 \\
\hline
\end{tabular}

\section{DISCUSSÃO E CONCLUSÕES}

0 presente artigo pretendia apresentar o modelo desenvolvido, no âmbito das Academias Gulbenkian do Conhecimento, para a replicação do programa de Aprendizagem SocioEmocional para $3^{\circ}$ ciclo.

Pelos resultados analisados, o modelo de formação parece ter uma satisfação elevada pelos participantes, fornecendo os conteúdos teóricos e práticos para a aplicação do programa nas respectivas Academias.

0 modelo de monitorização, composto pela plataforma e pelas visitas de acompanhamento, parece ter sido satisfatório para as academias, mas também permitiram reunir um conjunto de dados de variáveis de processo, indispensáveis para os estudos de efectividade que estão a ser desenvolvidos pela equipa.

Em suma, o modelo desenvolvido e apresentado no presente artigo, parece ter uma estrutura e duração adequada para a formação e monitorização das Academias. Estas conclusões, aliadas aos primeiros resultados da replicação national, publicada pelos autores (Coelho, Romão, Silva e Saldanha, 2021), confirmam a importância da formação e monitorização das equipas implementadoras dos programas. Considerando que os autores não encontraram diferenças significativas nos resultados, pela experiencia dos aplicadores, o modelo de formação e monitorização gana destaque para o sucesso das replicações de programas de Aprendizagem SocioEmocional.

\section{REFERÊNCIAS BIBLIOGRÁFICAS}

Collaborative for Academic, Social, and Emotional Learning (2012). 2013 CASEL guide: Effective social and emotional learning programs (Preschool and elementary school edition). Chicago, IL: Weissberg, R. P., Goren, P., Domitrovich, C., \& Dusenbury, L.

Coelho, V., Marchante, M. \& Sousa, V. (2015). "Positive Attitude": A multilevel model analysis of the efectiveness of a social and emotional learning program for Portuguese middle school students. Journal of Adolescence, 43, 29 38. https://doi.org/10.1016/j.adolescence.2015.05.009

Coelho, V., Marchante, M. \& Sousa, V. (2016). Positive Attitude program's impact upon self concept across childhood and adolescence. Revista de Psicodidactica, 21(2), 261-280. doi: 10.1387/RevPsicodidact.15129

Coelho, V. A., \& Sousa, V. (2017). The impact of class-level variables on the effectiveness of a middle school social and emotional learning program: A multilevel analysis. Journal of Relationships Research, 8, e21. doi: 10.1017/jrr.2017.21

Coelho, V., \& Sousa, V. (2018). Differential Effectiveness of a Middle School Social and Emotional 
Learning Program: Does Setting Matter? Journal of Youth and Adolescence, 47, 1978-1991. doi: 10.1007/s10964-018-0897-3

Coelho, V., Sousa, V. \& Figueira, A. P. (2014). The impact of a school based social and emotional learning program on the self concept of middle school students. Revista de Psicodidáctica, 19(2), 347 365. https://doi.org/10.1387/RevPsicodidact.10714

Coelho, V., Sousa, V., \& Marchante, M. (2014). Desenvolvimento e validação do questionário de avaliação de competências socioemocionais - versão professores [Development and validation of the Social and Emotional Competencies Questionnaires - Teacher's version]. International Journal of Developmental and Educational Psychology,2(1), 15-22.

Coelho, V., Sousa, V., \& Marchante, M. (2015). Development and validation of the social and emotional competencies evaluation questionnaire. Journal of Developmental and Educational Psychology, 5, 139-147. doi:10.5539/jedp.v5n1p139

Coelho, V. A., Sousa, V., \& Marchante, M. (2016). Social and Emotional Competencies Evaluation Questionnaire-Teacher's Version: Validation of a Short Form. Psychological Reports, 119(1), 221-236. https://doi.org/10.1177/0033294116656617

Coelho, V. A., \& Sousa, V. (2016). Desenvolvimento de um sistema de avaliação multi-informantes de competências socioemocionais. In A. M. Pinto \& R. Raimundo (Eds.) , Avaliação e promoção de competências socioemocionais em Portugal (pp. 135-153). Lisboa: Coisas de Ler

Coelho, V. A., Sousa, V., Raimundo, R., \& Figueira, A. (2015). The impact of a Portuguese middle school social-emotional learning program. Health Promotion International, 32, 292-300.

Coelho, V. A., Romão, A. M., Silva, P. R., Saldanha, S. (2021). Un análisis a nivel nacional de la efectividad de un programa de aprendizaje socioemocional en Portugal: enfoque sobre el papel de la participación de los creadores. Revista Psicodidáctica, online first. https://doi.org/10.1016/j.psicod.2021.04.001

Durlak, J., Weissberg, R., Dymnicki, A., Taylor, R. \& Schellinger, K. (2011). The impact of enhancing students' social and emotional learning: A meta-analysis of school-based universal interventions. Child Development, 82, 405-432. doi: 10.1111/j.1467-8624.2010.01564.x

Fontaine, A. M. (1991). Desenvolvimento do conceito de si próprio e realização escolar na adolescência. Psychologica, 5, 1331.

Greenberg, M. T., Domitrovich, C. E., Weissberg, R. P., Durlak, J. A. (2017). Social and Emotional Learning as a Public Health Approach to Education. Future of Children, 27(1), 13-32.

Knapp M., McDaid D., Parsonage M. (2011) Mental Health Promotion and Mental IIIness Prevention: The Economic Case. Department of Health, London.

Sklad, M., Diekstra, R., DeRitter, M., Ben, J. \& Gravesteijn, C. (2012). Effectiveness of school-based universal social, emotional, and behavioral programs: Do they enhance students' development in the area of skill, behavior and adjustment? Psychology in the Schools, 49, 892-909. doi: $10.1002 /$ pits. 21641

Taylor, R. D., Oberle, E., Durlak, J. A., \& Weissberg, R. P. (2017). Promoting positive youth development through school-based social and emotional learning interventions: A meta-analysis of follow up effects. Child Development, 88, 1156-1171. doi:10.1111/cdev.12864.

Weissberg, R. P., Durlak, J. a, Domitrovich, C. E., \& Gullotta, T. P. (2015). Social and Emotional Learning: Past, present, and future. Handbook of Social and Emotional Learning: Research and Practice, (July), 3-19. 
